\title{
DETERMINACIÓN DE LA CORRELACIÓN EN EL USO DEL CUESTIONARIO DE VANDERBILT Y LA APLICACIÓN DE PRUEBAS NEUROPSICOLÓGICAS PARA EL DIAGNÓSTICO DEL TRASTORNO POR DÉFICIT ATENCIONAL.
}

\author{
DETERMINATION OF THE CORRELATION IN THE USE OF THE VANDERBILT QUESTIONNAIRE AND THE \\ APPLICATION OF NEUROPSYCHOLOGICAL TESTS FOR THE DIAGNOSIS OF THE ATTENTIONAL DEFICIT \\ DISORDER \\ Carreño Álvarez Miguel*, Gatica Ferrero Sergio*. \\ * Facultad de Educación de la Universidad Católica de la Santísima Concepción, Chile.
}

Citation: Carreño Álvarez M., Gatica Ferrero S. (2019) Determinación de la correlación en el uso del Cuestionario de Vanderbilt y la aplicación de pruebas neuropsicológicas para el diagnóstico del Trastorno por Déficit Atencional. Revista de Salud Pública y Nutrición, 18 (2), 1-7.

Editor: Esteban G. Ramos Peña, Dr. CS., Universidad Autónoma de Nuevo León, Facultad de Salud Pública y Nutrición, Monterrey Nuevo León, México. Copyright: (C2019 Carreño Álvarez M. et al. This is an open-access article distributed under the terms of Creative Commons Attribution License [CC BY 4.0], which permits unrestricted use, distribution, and reproduction in any medium, provided the original author and source are credited.

Competing interests: The authors have declared that no competing interests exist.

DOI: https://doi.org/10.29105/respyn18.2-1

Recibido: 16 de enero 2019; $\quad$ Aceptado: 23 de mayo 2019

Email: sgatica@ucsc.cl 


\title{
DETERMINACIÓN DE LA CORRELACIÓN EN EL USO DEL CUESTIONARIO DE VANDERBILT Y LA APLICACIÓN DE PRUEBAS NEUROPSICOLÓGICAS PARA EL DIAGNÓSTICO DEL TRASTORNO POR DÉFICIT ATENCIONAL
}

\author{
Carreño Álvarez Miguel*, Gatica Ferrero Sergio*. \\ * Facultad de Educación de la Universidad Católica de la Santísima Concepción, Chile.
}

\section{RESUMEN}

Introducción. El trastorno por déficit atencional con hiperactividad (TDAH) es el trastorno del neurodesarrollo más habitual en la investigación y práctica clínica con escolares. Se caracteriza por síntomas de inatención y de hiperactividad/impulsividad que dificultan el aprendizaje y la adaptación positiva a la escuela. El TDAH suele ser detectado por medio de escalas de observación del comportamiento y de test neuropsicológicos. El Decreto 170/2009 del Ministerio de Educación chileno plantea un protocolo de detección basado en la aplicación del Cuestionario Abreviado Conners sin contemplar los aspectos cognitivos que aportan los test neuropsicológicos. Objetivo: Determinar el grado de correlación entre un cuestionario de observación de conducta y los resultados de una batería neuropsicológica de evaluación de la atención y la inhibición. Material y Métodos: Se evaluaron 70 alumnos de Educación Básica chilenos con el NICHQ Vanderbilt Assessment Scale y con tests neuropsicológicos para atención e inhibición; los resultados fueron analizados con $r$ de Pearson. Resultados: No existe correlación entre los puntajes obtenidos con NICHQ y los test neuropsicológicos. Conclusiones: Las escalas de observación del comportamiento y los test neuropsicológicos no pueden ser utilizados unos en reemplazo de otros, más bien parecen constituir un continuo dentro de la evaluación del TDAH.

Palabras Clave: Trastornos del aprendizaje, DSM-V, cuestionarios de observación del comportamiento, evaluación neuropsicológica, funciones ejecutivas.

\section{ABSTRACT}

Introduction: Attention-deficit hyperactivity disorder (ADHD) is the most common neurodevelopmental disorder in research and clinical practice with schoolchildren. It is characterized by symptoms of inattention and hyperactivity / impulsivity that hinder learning and positive adaptation to school. ADHD is usually detected through behavioral observation scales and neuropsychological tests. Decree 170/2009 of the Chilean Ministry of Education proposes a detection protocol based on the application of the Conners Abbreviated Questionnaire without considering the cognitive aspects that neuropsychological tests provide. Objective: To determine the degree of correlation between a behavioral observation questionnaire and the results of a neuropsychological battery of evaluation of attention and inhibition. Methods: 70 Chilean Basic Education students were evaluated with the NICHQ Vanderbilt Assessment Scale and with neuropsychological tests for attention and inhibition; the results were analyzed with Pearson's r. Results: There is no correlation between the scores obtained with NICHQ and the neuropsychological tests. Conclusions: Behavioral observation scales and neuropsychological tests cannot be used to replace others, rather they seem to be a continuum in the evaluation of ADHD.

Key words: Learning disorders, DSM-V, behavioral observation questionnaires, neuropsychological evaluation, executive functions. 


\section{Introducción}

El trastorno por déficit de atención con hiperactividad (TDAH) es el trastorno del neurodesarrollo más habitual en la investigación y la práctica clínica de niños en edad escolar (Salas, González, Araya, Valencia y Oyarce, 2017) con una alta prevalencia en la población infantil que varía entre un 5 a 10\% (Fernández, López, Albert, Fernández, Fernández, Calleja y López, 2017). Se considera como un trastorno del neurodesarrollo en base a la clasificación prescrita por el DSM-V, cuyos síntomas nucleares son dificultades a nivel atencional y signos de hiperactividad e impulsividad, dando lugar a tres subtipos de TDAH, (1) con predominio inatento, (2) con predominio hiperactivo/impulsivo y (3) con predominio combinado. Se ha podido observar que el TDAH tiene un fuerte componente disejecutivo, manifestado en alteraciones la atención (con sus redes de arousal, orientación y control ejecutivo), la inhibición de respuestas automáticas y en medida variable, la capacidad de planificación, memoria de trabajo, entre otros (Petersen \& Posner, 2012).

La detección del TDAH se lleva a cabo en base a la evaluación neuropsicológica y a la observación conductual. La primera corresponde al desempeño que alcanza el sujeto en test de características psicométricas de lápiz y papel que evalúan, principalmente, la atención y el control de impulsos. La segunda corresponde a la aplicación de cuestionarios de observación conductual, aplicados por padres y profesores. Existe un amplio debate relativo a la pertinencia de los cuestionarios y las escalas de observación conductual para la detección de TDAH, debido a la longitud de la lista de síntomas incluidos en el DSM-V (Barkley, 2009).

En Chile, el Decreto 170/2009 (2010) del Ministerio de Educación fija las normas para determinar a los alumnos con necesidades educativas especiales que serán beneficiarios de las subvenciones para educación especial. El documento explicita los procedimientos para la detección y derivación para la evaluación diagnóstica integral del TDAH, entre los que se incluyen la utilización de pruebas en basadas en criterios como el Test de Conners. Son mencionados también el Cuestionario de Observación del Comportamiento en la Sala de Clases para Profesores (TOCA-RR) y el Cuestionario para Padres PSC. No se hace alusión al uso de instrumentos neuropsicológicos que puedan limitar el efecto del sesgo del evaluador.

Algunos de los cuestionarios con mayor difusión para la evaluación del TDAH son (a) el Test de Conners, (b) la Escala para la Evaluación del Trastorno por Déficit de Atención con Hiperactividad (EDAH), (c) el Cuestionario para Padres y Maestros NICHQ Vanderbilt Assessment Scale, y (d) el ADHD Self-Report Scale.

La evaluación neuropsicológica del TDAH suele utilizar algunos test de manera más frecuente, tales como (a) Prueba de Memoria de Trabajo de WISCIV, (b) la Prueba Continuous Performances Test (CPT), (c) el Test de los Cinco Dígitos (FDT), (d) el Test de Stroop, y (e) el Test de Percepción de Diferencias-Revisado (CARAS-R).

En Chile los cuestionarios sobre atención y conducta tienen un uso más extendido que las pruebas neuropsicológicas, por lo que tienen un lugar preponderante en el reconocimiento del TDAH. Esto va en abierta contradicción con la investigación actual, la cual sostiene que las evaluaciones neuropsicológicas vienen a complementar el uso de cuestionarios y de los criterios diagnósticos establecidos en el DSM-V y permiten establecer una línea de base para la posterior intervención (AbadMas, Caloca-Català, Mulas y Ruiz-Andrés, 2017). Un estudio realizado en México observó que al aplicar pruebas psicométricas como la Figura de Rey-Osterrieth y la prueba de Cancelación Visual de Mesalum, los resultados obtenidos por los alumnos no se correlacionaron de manera significativa con los alcanzados por medio de la aplicación de encuesta de Conners para profesores (Flores-Aréchiga, GarzaGonzález, Llaca-Díaz y Gómez-Espinel, 2016). Resultados semejantes obtuvieron Young \& Gudjonsson (2005), quienes utilizaron el Young ADHD Questionnaire-I (YAQ-I), cuestionario auto informado, y Young ADHD Questionnaire-S (YAQ$S)$, cuestionario informado por terceros, aplicado por examinadores entrenados. Paralelamente se aplicó a los sujetos una batería neuropsicológica conformada por el Letter Cancelation Test (LCT), Continuos Performance Test (CPT) y Matching Familiar Figures test (MFF). Los investigadores concluyeron que las pruebas neuropsicológicas no se correlacionan necesariamente con los cuestionarios aplicados y que incluso, el sólo uso de pruebas neuropsicológicas no bastaría para establecer un 
diagnóstico diferencial entre el TDAH y otras alteraciones psiquiátricas.

Los cuestionarios presentan una clara ventaja en su aplicación en las escuelas; pueden ser rápidamente aplicados por profesores y su coste es bajo. Sin embargo, presentan altos niveles de discrepancia al ser comparados los reportes de docentes y familiares (Salas, González, Araya, Valencia, \& Oyarce, 2017). Esto no ocurre con las pruebas neuropsicológicas, cuyos resultados son consistentes entre diferentes evaluadores; sin embargo, su uso es más limitado por factores económicos y de especialización de examinadores. No obstante estas observaciones, otros autores utilizan de modo exitoso escalas y cuestionarios de comportamiento para la detección del TDAH en investigación, utilizando los criterios del DSM-IV y DSM-V (Barkley, 2009; Becker, 2013), la escala Conners (Sims, Purpura \& Lonigan, 2012), la Child and Adolescent Disruptive Behavior Inventory (CADBI) (Lee, Burns, Snell y McBurnett, 2013) o la Vanderbilt ADHD Diagnostic Teacher Rating Scale (Langberg, Vaughn, Brinkman, Froehlich, \& Epstein, 2010; Becker, 2013). Los autores suelen referir un proceso de entrenamiento previo en el uso de cuestionarios y escalas de observación del comportamiento que otorgan solidez a la detección de TDAH con estos instrumentos.

En base a lo anterior, resulta interesante conocer si en el contexto de una escuela pública de la región del Biobío en Chile, las escalas y cuestionarios de observación del comportamiento pueden utilizarse en lugar de test neuropsicológicos para la detección del TDAH, tal como sugiere el Decreto 170/2009 del Ministerio de Educación. Por lo tanto, el objetivo del estudio es determinar el grado de correlación entre los resultados del Sistema NICHQ Vanderbilt de Evaluación (cuestionario del profesor), basado en los criterios diagnósticos del DSM-V y los resultados de una batería neuropsicológica de evaluación de la atención y la inhibición.

\section{Material y Métodos}

Tamaño y selección de la muestra

La muestra estuvo constituida por 75 alumnos de tres cursos diferentes pertenecientes al ciclo de Enseñanza General Básica (EGB) de una escuela pública de la comuna de Coronel, región del Biobío, Chile. Se utilizó un muestreo de tipo no probabilístico por conveniencia en base a la propuesta de muestreo establecida por Blaxter, Hughes y Tight (2002). Se excluyen del estudio cinco alumnos que presentan trastorno del espectro autista, capacidad intelectual límite y discapacidad intelectual (Manterola y Otzen, 2015), debidamente acreditadas por profesionales afines (psicólogo, neurólogo, psiquiatra), por lo que la cantidad total de sujetos participantes se reduce a 70 . Ocho niños de la muestra contaban con diagnóstico previo de TDAH, situación que no era conocida por los informantes. Se evaluó a niños y niñas de $3^{\circ}(\mathrm{n}=23), 4^{\circ}(\mathrm{n}=27)$ y $8^{\circ}$ año $(n=20)$ de Enseñanza General Básica (EGB), con una edad promedio de 138,8 meses (11,5 años). Un $45,71 \%$ de la muestra eran niñas $(n=32)$ y un $54,28 \%$ ( $\mathrm{n}=38$ eran niños).

Todos los profesores, apoderados y estudiantes aceptaron voluntariamente participar del estudio. En el caso de los alumnos, sus padres/apoderados debieron firmar un consentimiento informado en que declaraban comprender la naturaleza y objetivos de la investigación y los derechos que les asisten en estos casos; los profesores debieron firmar un documento similar. En todo el proceso evaluativo se respetaron las normas éticas de investigación con personas contenidas en la Declaración de Helsinki.

\section{Instrumentos de recolección de información}

Cuestionario NICHQ Vanderbilt Assessment Scale (NICHQ). Es un cuestionario de screening para la detección del TDAH, trastorno oposicionista desafiante (TOD), trastorno di-social (TD), ansiedad/depresión (A/D) y desempeño/participación escolar (DPE). Para el presente estudio sólo se utilizó el screening para TDAH compuesto por 18 indicadores, 9 para inatención y 9 para hiperactividad, correspondientes a los mencionados por el DSM-V. Se trata de una escala que permite graduar las conductas según su frecuencia. Se hace uso de su versión traducida, la que ha sido usada en otros estudios (García-Noriega y Bárcena-Calvo, 2013). Es informado por los profesores de cada participante.

Test de Percepción de Diferencias-Revisado (CARAS-R). Evalúa la atención de los sujetos mediante la capacidad de determinar diferencias entre figuras semejantes (Monteoliva, Carrada, \& Ison, 2017). Consta de 60 ítems, compuestos por tres dibujos esquemáticos de caras, de las cuales una se diferencia del resto en uno o más rasgos (cabello, 
cejas y boca). El sujeto debe seleccionar la cara diferente y dispone de tres minutos para completar la mayor cantidad de ejercicios posible. Se utiliza con sujetos entre los 6 y 18 años; su aplicación puede hacerse individual o colectiva. Su fiabilidad total obtenida con $\alpha$ de Cronbach es de 0,91 y está baremada en percentiles y eneatipos por curso.

Índice de Atención/Concentración. Corresponde a un índice complementario del Test de Memoria y Aprendizaje (TOMAL). El TOMAL permite evaluar memoria, aprendizaje, atención, entre otros. De esta prueba se hace uso del índice de Atención/Concentración, que está compuesto por los subtest de: a) dígitos en orden directo, b) dígitos en orden indirecto, c) letras en orden directo, d) letras en orden indirecto, y e) imitación manual. Los subtest utilizados son pruebas clásicas de span de dígitos directo e inverso profusamente utilizados en test de inteligencia. Se aplica a sujetos entre los 5 y los 19 años de edad de forma individual. Su fiabilidad, de 0,98, fue obtenida mediante $\alpha$ de Cronbach $\mathrm{y}$ entrega puntajes baremados en percentiles y puntuaciones escalares.

Test de los cinco dígitos (FDT). Evalúa la capacidad de control inhibitorio y flexibilidad cognitiva. Cada una de las cuatro tareas que la componen, consta de una página que contiene 50 estímulos, dispuestos en 10 filas de 5 ítems cada una. Cada ítem a su vez, se compone de cinco signos que pueden ser dígitos o asteriscos, con los cuales el sujeto debe realizar cuatro diferentes operaciones: a) parte de lectura; b) parte de conteo; c) parte de elección (contar independiente del valor del dígito y d) parte de alternancia (contar y leer dígitos, según corresponda). Se utiliza con sujetos de 7 años en adelante; su aplicación es individual. En el presente estudio se ha utilizado solamente para medir la interferencia o inhibición. Su fiabilidad fue obtenida con el procedimiento de las dos mitades, y corregidos posteriormente los índices con la fórmula de Spearman-Brown con los valores siguientes, lectura 0,95 , conteo 0,94 , elección 0,93 y alternancia 0,92 . El FDT fue baremado en percentiles.

Aplicación de los instrumentos.

La aplicación de la Escala NICHQ fue realizada por profesores que trabajan directamente con los alumnos evaluados. Para todos los casos (niños de $3^{\circ}$, $4^{\circ}$ y $8^{\circ}$ de EGB) se consideró a dos informantes por alumno; el primero correspondía a un profesor de aula, preferentemente de las asignaturas de Lenguaje y Comunicación o Educación Matemática; el segundo correspondía a una educadora diferencial. Los profesores informantes $(n=6)$ realizaron una capacitación de 16 horas en los fundamentos teóricos y aplicaciones prácticas del NICHQ Vanderbilt Assessment Scale - Teacher Informant, a cargo de un académico especialista de la Universidad Católica de la Santísima Concepción (UCSC), Chile. Cada par de informantes recopiló información conductual de cada estudiante durante dos semanas; pasado ese tiempo, y con asesoría del Coordinador del Programa de Inclusión Escolar de la escuela, la pareja de informantes completó el cuestionario NICHQ consensuando sus respuestas. Se realizó este procedimiento para disminuir el efecto de sesgo. Paralelamente, un equipo de tres evaluadoras de la Carrera de Educación Diferencial de la UCSC, aplicó los test neuropsicológicos a la muestra $(\mathrm{n}=70)$.

\section{Procedimientos}

Los procedimientos aplicados se dividieron en cuatro etapas, siendo éstas,

a) Consentimiento informado; tanto alumnos como padres/tutores deben aceptar participar.

b) Capacitación en aplicación de Cuestionario NICHQ para maestros

c) Aplicación de los instrumentos (cuestionario y baterías neuropsicológicas.

d) Interpretación de los resultados en base a procesamiento de datos.

Análisis de los resultados.

Los resultados fueron sometidos a la prueba de normalidad de Shapiro-Wilk (Hernández-Sampieri, Fernández-Collado y Baptista-Lucio, 2010) para determinar el estadígrafo de correlación más apropiado para el caso. El análisis permitió seleccionar el coeficiente de correlación de Pearson en base a la distribución normal de los datos. Para el procesamiento de datos, se utilizó el programa SPSS 24.0.

\section{Resultados}

Variable inatención. Se analizan los resultados obtenidos por medio de: a) Cuestionario NICHQ para inatención (VANI), b) índice de Atención/Concentración de Test TOMAL (IAC), y c) test de Percepción de Diferencias (CA). Los resultados se muestran en tabla 1 . 
Tabla 1. Correlaciones entre instrumentos que miden variable inatención.

\begin{tabular}{lcccc}
\hline & & VANI & IAC & CA \\
\hline VANI & Correlación de Pearson & 1 &,- 175 &,- 195 \\
IAC & Correlación de Pearson &,- 175 & 1 &, $252^{*}$ \\
CA & Correlación de Pearson &,- 195 &, $252^{*}$ & 1 \\
\hline *. La correlación es significativa en el nivel 0,05 (2 colas). & & $\mathrm{N}=70$ \\
Fuente: Elaboración propia & & &
\end{tabular}

El análisis de los resultados muestra ausencia de correlación estadísticamente significativa entre las variables principales representadas por el Cuestionario NICHQ para inatención, por un lado, y los resultados neuropsicológicos en atención representados por el rendimiento en IAC y el CA, por el otro. Por otra parte, tampoco se observa correlación significativa entre las baterías neuropsicológicas aplicadas.

Variable hiperactividad/impulsividad. Se analizan los resultados obtenidos por medio de: a) Cuestionario NICHQ para hiperactividad/impulsividad (VANHI), y b) Test de los Cinco Dígitos (FDT). Los resultados se muestran en la tabla 2.

Tabla 2. Correlaciones entre instrumentos que miden variable hiperactividad/impulsividad

\begin{tabular}{lccc}
\multicolumn{4}{c}{ hiperactividad/impulsividad } \\
\hline & VANHI & FDT \\
\hline VANHI & Correlación de Pearson & 1 &,- 060 \\
FDT & Correlación de Pearson &,- 060 & 1 \\
\hline Fuente: Elaboración propia & & $\mathrm{N}=70$
\end{tabular}

El análisis de los resultados muestra ausencia de correlación estadísticamente significativa entre las variables principales representadas por el Cuestionario NICHQ para hiperactividad/impulsividad, por un lado, y los resultados neuropsicológicos en atención representados por el rendimiento en el FDT.

Correlación entre baterías neuropsicológicas. Se analizan los resultados de a) IAC (atención), b) CARAS-R (atención), y c) inhibición (FDT). Los resultados se muestran en la tabla 3.

Tabla 3. Correlación entre los resultados obtenidos en pruebas neuropsicológicas

\begin{tabular}{llccc}
\hline & & FDT & IAC & CA \\
\hline FDT & Correlación de Pearson & 1 &, 160 &, 127 \\
IAC & Correlación de Pearson &, 160 & 1 &, $252^{*}$ \\
CA & Correlación de Pearson &, 127 &, $252^{*}$ & 1 \\
\hline *. La correlación es significativa en el nivel 0,05 (2 colas). & & $\mathrm{N}=70$
\end{tabular}

Fuente: Elaboración propia

El análisis de los resultados muestra que no existe una correlación significativa entre las baterías de atención (IAC y CA) y la de inhibición (FDT). En el caso particular de la correlación entre IAC y CA, existe menos de un 5\% de error en el grado de correlación obtenida, lo que nos asegura que esta correlación, aunque débil, es positiva.

Correlación interna entre subcomponentes de Cuestionario NICHQ. Se analizan los resultados obtenidos por los dos componentes del Cuestionario NICHQ para TDAH relativos a inatención e hiperactividad/impulsividad. Los resultados se muestran en la tabla 4.

\begin{tabular}{|c|c|c|c|}
\hline & & VANI & VANHI \\
\hline VANI & Correlación de Pearson & 1 &, $671 * *$ \\
\hline VANHI & Correlación de Pearson &, $671 * *$ & 1 \\
\hline
\end{tabular}

El análisis de los resultados muestra una correlación moderado significativa entre los componentes de la escala NICHQ.

\section{Discusión}

Los resultados obtenidos permiten observar que no existe correlación entre los resultados obtenidos mediante el Cuestionario NICHQ para maestros y las pruebas neuropsicológicas seleccionadas. Tres aspectos más son interesantes de notar. En primer lugar, las tareas de atención medidas con test neuropsicológicos correlacionan significativamente, cuestión que puede interpretarse en el sentido de que ambos test miden una habilidad ejecutiva común que no parece depender del input sensorial (visual para CARAS-R y auditivo para IAC). Dado que no es una correlación fuerte, sería interesante estudiar en qué medida un test puede reemplazar al otro, o si ambos deben ser utilizados complementariamente en una batería de exploración amplia.

En segundo lugar, la falta de correlación estadística entre los test de atención (CARAS-R e IAC) y el test de control inhibitorio refuerzan la idea, extendida en la literatura, que se tarea de dos funciones ejecutivas diferentes que deben ser abordadas por la evaluación neuropsicológica del TDAH. En esta línea AbadMas et al., (2017) señala que las pruebas neuropsicológicas pueden venir a complementar los criterios diagnósticos establecidos en DSM-V, los cuales considera como imprecisos. 
En tercer lugar, la significativa correlación entre las escalas de inatención e hiperactividad/impulsividad en el Cuestionario NICHQ parece indicar que, pudiendo ser una herramienta valiosa para la detección de TDAH, no parecen tener capacidad discriminatoria suficiente para diferenciar entre los subtipos del trastorno.

De esta forma, se obtienen resultados similares al estudio de Flores-Aréchiga, Garza-González, LLacaDíaz y Gómez Espinel (2016), en cuanto a que no existirían correlaciones significativas entre el uso de cuestionarios y baterías neuropsicológicas. Específicamente el uso de cuestionarios de observación conductual depende, en alguna medida del juicio del evaluador, cuestión que no suele ocurrir con el uso de test neuropsicológicos.

De la misma forma, Young \& Gudjonsson (2005) concluyeron que no existiría una correlación estadísticamente significativa entre los resultados obtenidos por medio de las dos metodologías de evaluación; en su estudio no desestiman el uso de cuestionarios ni el uso de baterías. Al igual que en nuestro estudio, señalan que el sólo uso de baterías no es suficiente para establecer un diagnóstico diferencial del TDAH. Además de eso, nuestros resultados parecen indicar que, pese a su uso habitual en investigación, el Cuestionario NICHQ no parece suficiente por sí mismo para detectar el TDAH en la población escolar chilena estudiada.

\section{Conclusiones}

Los resultados obtenidos por medio del uso del Cuestionario NICHQ para maestros y de pruebas neuropsicológicas aplicadas, permiten sugerir que ambas metodologías de evaluación no compiten entre sí, ni pueden ser usadas una en reemplazo de la otra. De hecho, ambos procedimientos parecen ser complementarios; permiten obtener una visión global del desempeño dentro del aula de clases (cuestionarios), así como medir rendimientos específicos en determinadas habilidades cognitivas (pruebas neuropsicológicas).

La falta de correlato entre ambas metodologías de evaluación para el TDAH, puede entenderse por los siguientes motivos: a) los criterios diagnósticos establecidos por DSM pueden ser insuficientemente claros, precisos y depurados (Abad-Mas, Caloca-
Català, Mulas \& Ruiz-Andrés, 2017), de hecho parecen tender a ser redundantes (Barkley, 2009); b) el grado de entrenamiento de los sujetos que informan los cuestionarios de conducta parece ser insuficiente, sobre todo al contrastar con las exitosas experiencias de otros autores; c) las pruebas neuropsicológicas evalúan habilidades medidas en rendimiento, mientras que los cuestionarios evalúan conductas en base a juicio de terceros (Hjern, Weitoft \& Lindblad, 2010; Weissenberger et al., 2017); y d) el rendimiento en pruebas neuropsicológicas puede deberse a alteraciones, trastornos o retrasos del desarrollo diferentes al TDAH.

Para efecto de los resultados obtenidos, puede entenderse que la aplicación de test neuropsicológicos resulta deseable para una mejor identificación de los sujetos, reduciendo el sesgo del evaluador (sobre todo de quienes evalúan mediante uso de cuestionarios) y aportando mayor información acerca de orientaciones psicoeducativas que permitan abordar las dificultades que presentan los alumnos.

\section{Limitaciones}

El análisis de los resultados ha permitido detectar dos limitaciones importantes en el estudio. La primera ha sido el limitado número de participantes, así como su poca representatividad socioeconómica, al tratarse de alumnos de un solo centro educativo; esta situación ha derivado en la imposibilidad de generalizar los resultados obtenidos. La segunda, ha sido no considerar el rendimiento escolar una variable en la investigación, a sabiendas que el TDAH cursa con un desempeño académico deficiente en diversas áreas del currículo escolar.

La observación de estas limitaciones nos orienta a resolverla en el futuro contando con una muestra más robusta e incluyendo el desempeño escolar como variable de estudio.

\section{Agradecimientos}

Esta investigación fue posible gracias al apoyo del Proyecto INDIN 04/2018 de la Universidad Católica de la Santísima Concepción del cual es investigador principal el segundo autor.

\section{Bibliografía}


Abad-Mas, L., Caloca-Català, O., Mulas, F., \& RuizAndrés, R. (2017). Comparación entre el diagnóstico del trastorno por déficit de atención / hiperactividad con el DSM-5 y la valoración neuropsicológica de las funciones ejecutivas. Rev Neurol, 64(Supl 1), 95-100.

Barkley, R. A. (2009). Avances en el diagnóstico y la subclasificación del trastorno por déficit de atención / hiperactividad : qué puede pasar en el futuro respecto al DSM-V. Rev Neurol, 48(Supl 2), 101-106.

Becker, S. P. (2013). Topical review: Sluggish cognitive tempo: research findings and relevance for pediatric psychology. Journal of Pediatric Psychology, 38(10), 1051-1057. https://doi.org/10.1093/jpepsy/jst058

Blaxter, L., Hughes, C., y Tight., M. (2002). Como se hace una Investigación. Barcelona: GEDISA Editorial.

Decreto 170/2009 (2010). Fija normas para determinar a los alumnos con necesidades educativas especiales que serán beneficiarios de las subvenciones para educación especial. Diario Oficial de la República de Chile.

Fernández, A., López, S., Albert, J., Fernández, Fernández L., Calleja, B., y López, S. (2017). Trastorno por déficit de atención / hiperactividad: perspectiva desde el neurodesarrollo, Rev Neurol, 64(Supl 1), 101-104.

Flores-Aréchiga, A., Garza-González, M., LLaca-Díaz, J., y Gómez Espinel, I. (2016). Comparación de cuatro instrumentos utilizados en la evaluación de los trastornos por deficit de atención e hiperactividad. Revista de Salud Pública y Nutrición, 15(2), 7.

García-Noriega, A. y Bárcena-Calvo, C. (2013). Valoración categorial del trastorno por déficit de atención/hiperactividad, mediante la escala de evaluación Vanderbilt en padres. Revista Enfermería Castilla y León, 5(1), 32-41.

Hernández-Sampieri, R., Fernández-Collado, C., y Baptista-Lucio, P. (2010). Metodología de la investigación. México D.F.: Mc Graw-Hill.

Hjern, A., Weitoft, G. R., \& Lindblad, F. (2010). Social adversity predicts ADHD-medication in school children - A national cohort study. Acta Paediatrica, International Journal of Paediatrics, 99(6), 920-924. https://doi.org/10.1111/j.1651-2227.2009.01638.x

Langberg, J. M., Vaughn, A. J., Brinkman, W. B., Froehlich, T., \& Epstein, J. N. (2010). Clinical utility of the Vanderbilt ADHD Rating Scale for ruling out comorbid learning disorders. Pediatrics, 126(5), e1033-e1038. https://doi.org/10.1542/peds.2010-1267
Lee, S., Burns, G. L., Snell, J., \& McBurnett, K. (2013). Validity of the Sluggish Cognitive Tempo Symptom Dimension in Children: Sluggish Cognitive Tempo and ADHD-Inattention as Distinct Symptom Dimensions. Journal of Abnormal Child Psychology, 42(1), 719.doi:10.1007/s10802-013-9714-3

Manterola, C., y Otzen, T. (2015). Los Sesgos en Investigación Clínica. International Journal of Morphology, 33(3), 1156-1164. https://doi.org/http://dx.doi.org/10.4067/S071795022015000300056

Monteoliva, J. M., Carrada, M., \& Ison, M. S. (2017). Test de percepción de diferencias: estudio normativo del desempeño atencional en escolares argentinos. Interdisciplinaria, 34(1), 39-56.

Petersen, S., \& Posner, M. (2012). The attention system of the human brain: 20 years after. Annual Review of Neuroscience, 21(35), 73-89. https://doi.org/10.1146/annurev-neuro-062111150525 .

Sims, D., Purpura, D. \& Lonigan. C., (2012). The relation between inattentive and hyperactive/impulsive behaviors and early mathematics skills. Journal of Attention Disorders 20(8), 704-714. doi:10.1177/1087054712464390

Salas, S., Gonzalez, M., Araya, A., Valencia, M., \& Oyarce, S. (2017). Uso del test de rendimiento continuo de conners para diferenciar niños normales y con TDAH en Chile. Terapia Psicologica, 35(3), 283 $291 . \quad$ https://doi.org/10.4067/S071848082017000300283

Weissenberger, S., Ptacek, R., Klicperova-Baker, M., Erman, A., Schonova, K., Raboch, J., \& Goetz, M. (2017). ADHD, lifestyles and comorbidities: A call for an holistic perspective - From medical to societal intervening factors. Frontiers in Psychology, 8 (MAR), 1-13. https://doi.org/10.3389/fpsyg.2017.00454

Young, S., \& Gudjonsson, G. H. (2005). Neuropsychological correlates of the YAQ-S and YAQ-I self- and informant-reported ADHD symptomatology, emotional and social problems and delinquent behaviour. British Journal of Clinical Psychology, 44(1), 47-57. https://doi.org/10.1348/014466504X197769. 